\title{
The Anderson-Winawer illusion: It's not occlusion
}

\author{
FrÉdÉRIC J. A. M. PoIRIER \\ Université de Montréal, Montréal, Québec, Canada
}

\begin{abstract}
In their recent article, Anderson and Winawer (2005) presented a dramatic lightness illusion in which identical texture patches appear to be either black or white. Albert (2007) argued that the Anderson and Winawer (2005) illusion can be explained by a simple theory in which occlusion cues determine the depth relationships of the different surfaces, and determine which stimulus areas are perceived as seen in plain view. Using both modeling and psychophysical methods, however, I show that alterations such as those that Albert used actually reverse the illusion within the range of figure contrasts that Anderson and Winawer (2005) tested. Albert's theory (and any occlusion-based theory), therefore, cannot account for Anderson and Winawer's (2005) data, at least in the lower figure-contrast range. I propose a novel scene-interpretation strategy to account for the effects.
\end{abstract}

In their recent article, Anderson and Winawer (2005) presented a dramatic lightness illusion in which identical patches appear to be either black or white. Figure 1A shows Anderson and Winawer's (2005) original stimuli, which perceptually segregate in three layers: a moon, a sky, and clouds. The moon (figure) and sky (background) are each perceived as homogeneously opaque with a uniform reflectance, whereas the clouds are perceived to have a uniform reflectance but variable opacity. As a result of this segregation, physically identical center regions appear as either white or black moons depending on the context (see, e.g., Figure 1, column 1), making this illusion much stronger than other known lightness illusions such as the White effect (White, 1979, 1981), simultaneous contrast (Heinemann, 1955; Horeman, 1963), and transparency effects (Adelson, 1993, 2000).

Anderson and Winawer's $(2005,2008)$ account for the effect can be decomposed in three parts: (1) detection of a variable-opacity occluder, (2) segregation of the occluder into a separate layer, and (3) lightness estimation.

In the first part of this model, changes in local edge contrast around the moon indicate the presence of a variable-opacity occluder. That is, the parts of the circle's edge where contrast is high are perceived as unoccluded, and the parts of the circle's edge where contrast is low are perceived as occluded. In the second part, the perceptual system segregates the occluder into a separate layer. In the third part, the figure's lightness is then determined on the basis of the properties of the center and surround areas. Specifically, Anderson and Winawer $(2005,2008)$ proposed that, independent of center contrast (see Figure 1, columns 1-6), figures that were lighter (or darker) than their surround were perceived as having a lightness that was equal to their lightest (or darkest) pixel. That is,
Anderson and Winawer's $(2005,2008)$ theory predicts that all moons in the upper row of Figure 1A will appear darker than all moons in the lower row, consistent with their results. As detailed below, the first and third parts of Anderson and Winawer's $(2005,2008)$ model produce different predictions in some conditions; hence, they cannot be equated.

Albert (2007) proposed a more detailed theory, the core of which relies on occlusion cues. In this respect, Albert's theory is equivalent to the first part of Anderson and Winawer's $(2005,2008)$ model. For simplicity, I will henceforth refer to two models: (1) the occlusion model, which refers to both Albert's model and the first part of Anderson and Winawer's $(2005,2008)$ model, and (2) the Anderson-Winawer prediction, which specifically refers to the third part of Anderson and Winawer's $(2005,2008)$ model, their model's output.

Albert (2007) used stimuli that were similar to those that are presented in Figure 1C (column 1) to argue that occlusion (see Anderson, 1997; Fine, MacLeod, \& Boynton, 2003; Kelly \& Grossberg, 2000; Li, 2000) is sufficient to generate the illusion. He provided examples in which the textures were simplified. That is, he removed extraneous information from the textures, so that only occlusion cues remained. He then generalized the occlusion mechanism to Anderson and Winawer's $(2005,2008)$ cloudy textures, in which sharp occlusion cues were replaced by blurred occlusion cues.

The occlusion model thus generates the same predictions regardless of whether the texture that is used is a square-wave grating (as Albert [2007] used to support his theory) or a cloudy texture (as was used by Anderson and Winawer [2005, 2008]). That is, Albert treated both textures as equivalent. Similarly, the occlusion model also

F. J. A. M. Poirier, frederic.poirier@umontreal.ca 


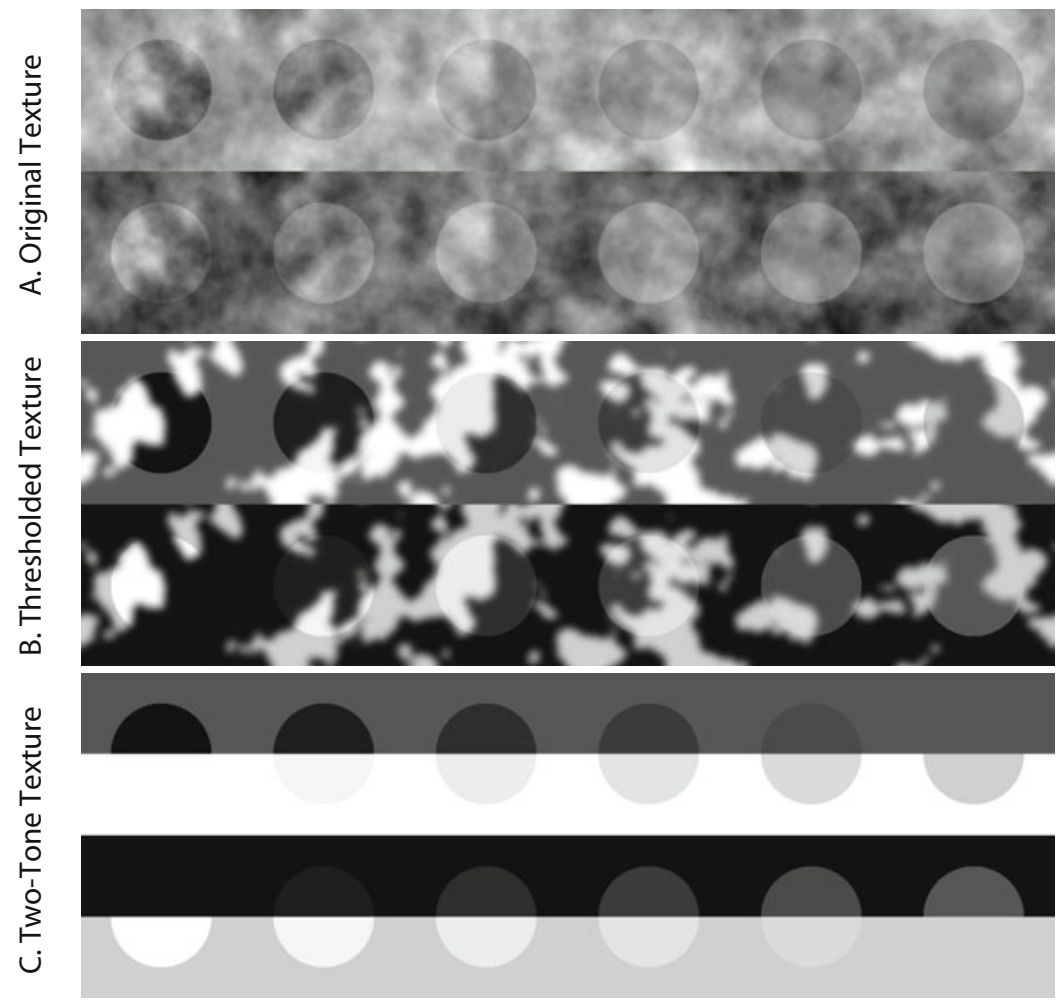

Figure 1. The Anderson-Winawer (2005) luminance configurations applied to the three different textures, with center contrast varying from high-contrast (left) to lowcontrast (right) figures. Surround mean luminance could be lighter (top of each row) or darker (bottom of each row) than center mean luminance. The cloudy texture (A) and two-tone texture $(\mathrm{C})$ were also used as stimuli in the present experiment. The texture change creates a drastic change in scene interpretation. Scene interpretation in Anderson and Winawer's (2005) cloudy textures (A) is independent of center contrast: We perceive a uniformly colored moon, with lightness equal to its darkest (or lightest) pixel when the center texture is on average darker (or lighter) than the surround texture. Scene interpretation in two-tone textures $(\mathrm{C}$; similar to the textures that were used by Albert [2007]) is clearly dependent on figure contrast. The visible part changes from the black top half (column 1) to the light-gray bottom half (column 6) for lighter surrounds (top row), and from the white bottom half (column 1) to the dark-gray top half (column 6) for darker surrounds (bottom row). Intermediate figure-contrast stimuli can be perceived as either a light or dark moon, or as a semitransparent dark disk floating over a textured background (columns 2-5; percept may not occur at all figure contrasts). Another way to describe the interaction of texture and luminance configuration is to decide which moon is lighter, the top or the bottom. In the stimuli with cloudy textures (A), the bottom moon is always lighter, whereas in the stimuli with two-tone textures $(\mathrm{C})$, the lighter moon is on the bottom row in column 1 but in the top row in column 6. This reversal also affects lightness of the occluder and of the background. The stimulus that is shown in (B) was built with the cloudy texture that was used in (A), except that it was thresholded and slightly blurred (to remove aliasing). The resulting stimulus is sometimes perceived consistently with the percept associated with the stimulus that is shown in $(\mathrm{A})$, and sometimes with the stimulus that is shown in $(\mathrm{C})$, and this percept switch can even occur within a trial.

generalizes to a two-tone texture (Figure 1C), a thresholded texture (Figure 1B), or any other texture with enough low-spatial-frequency content.

Albert (2007) argued that nearby parts of the center and surround regions that match in luminance are perceived as belonging to the clouds (thus occluding the moon and sky), whereas parts that differ in luminance appear as unoccluded by clouds (thus revealing the moon and sky). The perceived luminance of the moon and sky are thus taken from unoccluded parts of the stimuli. For example, accord- ing to the occlusion model, the moon is perceived as black in the top row of Figure 1C because the black top half of the circle is visible (i.e., unoccluded), whereas the moon is perceived as white in the bottom row of Figure $1 \mathrm{C}$ because the white lower half of the circle is visible (i.e., unoccluded). Albert argued, using the stimulus in column 1 of Figure $1 \mathrm{C}$, that there is nothing illusory in these percepts. Additional components of Albert's theory, such as anchoring, do not affect the conclusions of the present article; therefore, they will not be discussed here. 
Table 1

Luminance Configurations, Defined As the Luminance Ranges $\left(\mathrm{cd} / \mathrm{m}^{2}\right)$ in the Center $\left(C_{\mathrm{A}}, C_{\mathrm{B}}\right)$ and Surround $\left(S_{\mathrm{A}}, S_{\mathrm{B}}\right)$ Regions

\begin{tabular}{lcrrrrrr}
\hline $\begin{array}{c}\text { Center } \\
\text { Contrast }\end{array}$ & Surround & $C_{\mathrm{A}}$ & $C_{\mathrm{B}}$ & $S_{\mathrm{A}}$ & $S_{\mathrm{B}}$ & $\mathrm{AW}$ & Occ \\
\hline High & Light & 6 & 99 & 15 & 99 & 6 & 6 \\
High & Dark & 6 & 99 & 6 & 61 & 99 & 99 \\
Low & Light & 15 & 61 & 15 & 99 & $\mathbf{1 5}$ & $\mathbf{6 1}$ \\
Low & Dark & 15 & 61 & 6 & 61 & $\mathbf{6 1}$ & $\mathbf{1 5}$ \\
\hline
\end{tabular}

Note-Also shown are predictions that were derived from the Anderson and Winawer model (AW) and the occlusion model (Occ). The two models produce inconsistent predictions for stimuli with low-contrast centers (highlighted in bold). The luminance difference at the center-surround edge varies from $C_{\mathrm{A}}-S_{\mathrm{A}}$ to $C_{\mathrm{B}}-S_{\mathrm{B}}$.

This article uses modeling and psychophysical methods to investigate the Anderson-Winawer $(2005,2008)$ illusion. In the Models section, I show that predictions that can be derived from the Anderson and Winawer model and those that can be derived from the occlusion model differ from each other significantly within the range of stimuli that were used by Anderson and Winawer (2005).

In the Results section, I report data for both the cloudy and the two-tone textures. Data from cloudy textures were consistent with the Anderson and Winawer model, whereas data from the two-tone textures were consistent with the occlusion model. That is, there was an interaction between texture and luminance configuration that neither model can fully account for. Thresholded textures were not included, since there is some ambiguity regarding their classification, and, as such, they do not provide a good test of either theory (see details below).

\section{Models}

Anderson and Winawer (2005) used four luminance configurations that produced stimuli for which the occluder reached $100 \%$ opacity in certain places. These are luminance configurations for which, in some places, the maximum (or minimum) luminance in the center exactly matches the maximum (or minimum) luminance in close proximity in the surround. These four luminance configurations are summarized in Table 1, along with predictions from the Anderson and Winawer model and the occlusion model. These four luminance configurations were also used to generate predictions in the models, and to generate stimuli in the psychophysical experiment.

Predictions for the two models. The predictions for the Anderson and Winawer model were derived using Anderson and Winawer's $(2005,2008)$ own rule, from part 3 of their model, which states that centers that are lighter (or darker) than their surround are perceived as having a lightness that is equal to their lightest (or darkest) pixel. They did not provide a standard by which to compare center and surround luminance, so I compared midrange luminance for simplicity. Their rule is thus mathematically defined as

$$
\mathrm{AW}=\left\{\begin{array}{l}
\max \left(C_{\mathrm{A}}, C_{\mathrm{B}}\right) \text { if } \frac{\left(C_{\mathrm{A}}+C_{\mathrm{B}}\right)}{2}>\frac{\left(S_{\mathrm{A}}+S_{\mathrm{B}}\right)}{2}, \\
\min \left(C_{\mathrm{A}}, C_{\mathrm{B}}\right) \text { otherwise, }
\end{array}\right.
$$

where the luminance range contained in the center is given by $\left[C_{\mathrm{A}} \ldots C_{\mathrm{B}}\right]$ and the luminance range in the surround is given by $\left[S_{\mathrm{A}} \ldots S_{\mathrm{B}}\right]$. Predictions using this rule are shown in Table 1, and in Figure 2 along with the data for the cloudy texture. Specifically, these predictions show a small decrease in effect size when figure contrast is lowered, but the effect's direction is maintained.

The predictions for the occlusion model are defined by the luminance that does not belong to the occluder. Both Albert (2007) and Anderson and Winawer (2005, 2008) agreed that the occluder is perceived at the location where the center luminance equals the surround luminance (i.e., either $C_{\mathrm{A}}=S_{\mathrm{A}}$ or $C_{\mathrm{B}}=S_{\mathrm{B}}$ ). According to the occluder model, therefore, the figure luminance is defined as the luminance that does not belong to the occluder. This is mathematically equivalent to

$$
\text { Occ }=\left\{\begin{array}{lll}
C_{\mathrm{A}} & \text { if } & C_{\mathrm{B}}=S_{\mathrm{B}} \\
C_{\mathrm{B}} & \text { if } & C_{\mathrm{A}}=S_{\mathrm{A}}
\end{array}\right.
$$

within the range of conditions that are tested in the present article. (It is unclear how the occluder mechanism would deal with situations in which $C_{\mathrm{A}} \neq S_{\mathrm{A}}$ and $C_{\mathrm{B}} \neq S_{\mathrm{B}}$.) Predictions using this rule are shown in Table 1 and in Figure 2, along with the data for the two-tone texture. Specifi-

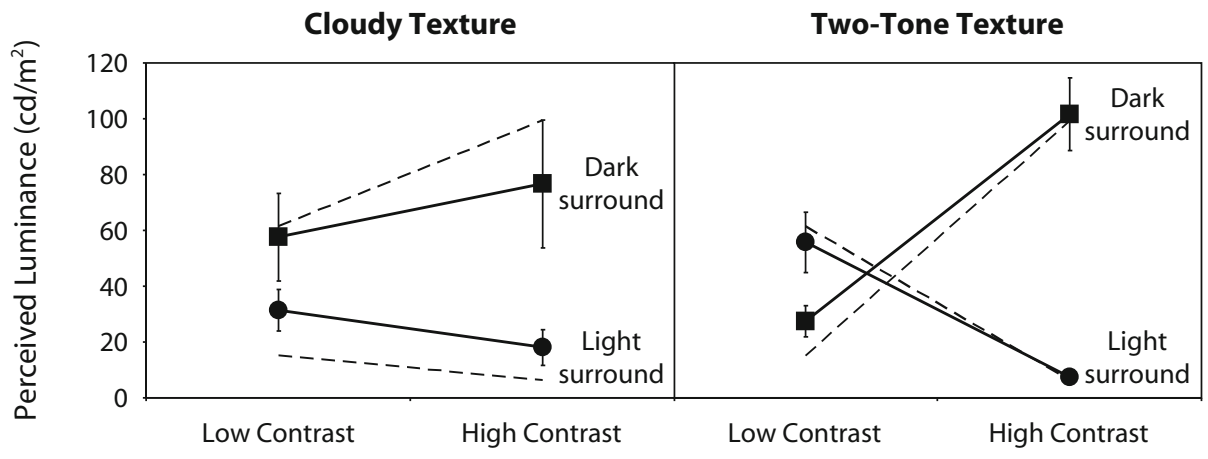

Figure 2. Perceived luminance as a function of texture (cloudy, two-tone), figure contrast (low, high), and surround luminance (dark, light). Also shown are model predictions (dashed lines). The effect of surround luminance reverses only for the two-tone textures with the low-contrast figures, as was predicted by the models. 
cally, these predictions show a small decrease in absolute effect size as well as a reversal in the effect's direction when figure contrast is lowered.

Comparison of model predictions. The two models generate the same prediction for stimuli with high-contrast centers (e.g., Figures $1 \mathrm{~A}-1 \mathrm{C}$, column 1) - that is, they both predict that the figure will be seen as white (or black) when the high-contrast center is placed on a dark (or light) surround.

The two models produce very different predictions for stimuli with low-contrast centers (e.g., Figures 1A-1C, column 6), however. This occurs because these stimuli (which were included in Anderson and Winawer's [2005] experiment) have the unique property of being, on average, lighter (or darker) than their surrounds yet generating occlusion cues that are consistent with a light (or dark) occluder. The Anderson and Winawer model predicts no reversal of the effect at lower center contrast, because as the center changes from high to low contrast, the center remains lighter (or darker) than its dark (or light) surround. The occlusion model predicts a reversal of the effect at lower center contrast, however, because the occlusion cues change from indicating the presence of black (or white) clouds to indicating light gray (or dark gray) clouds on dark (or light) surrounds.

Figure 3 is a concrete example of a stimulus with low contrast. In this example, participants reported that

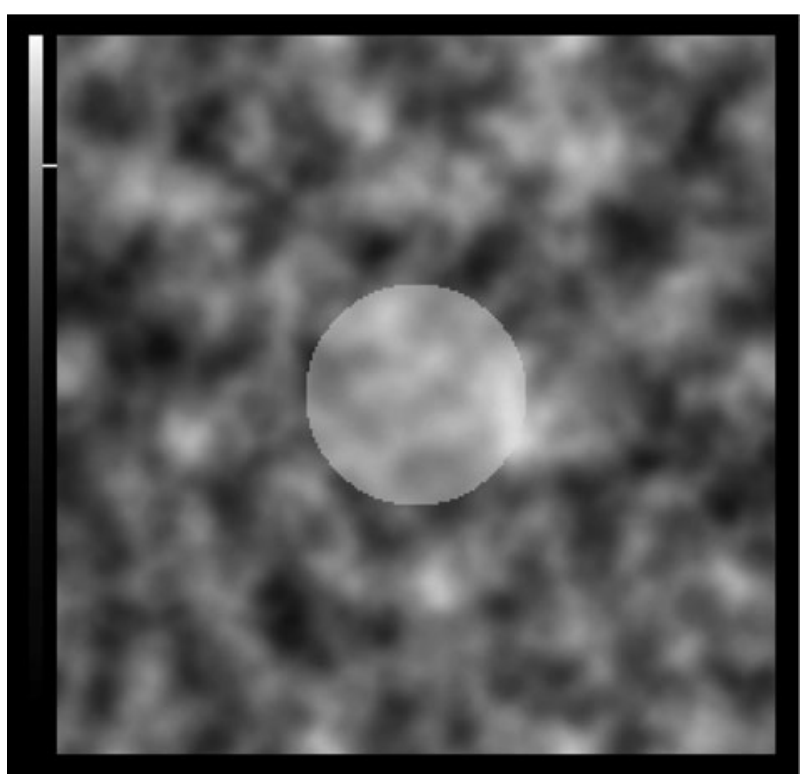

Figure 3. A sample stimulus as it was presented during the experiment, corresponding to the cloudy texture with one of the Anderson-Winawer (2005) luminance configurations (low center contrast, dark surround). Participants indicated the perceived luminance(s) on the white-to-black bar on the left. A small white bar indicated their response, which they could adjust before passing to the next stimulus. In this example, the participant reported perceiving the circle as light gray. For this stimulus, the Anderson and Winawer (2005) model predicts that participants will perceive the moon as light gray, whereas the occlusion model predicts that participants will perceive the moon as dark gray. This apparent contradiction occurs because the cues are conflicting. the moon was light gray. Indeed, consistent with the Anderson-Winawer prediction, the center is lighter than the surround on average; thus, the lightest pixel (light gray) was chosen as reflecting the moon's true luminance. According to the occlusion model, however, that pixel belongs to an area that is classified as belonging to the occluder. Indeed, the lightest pixel of the center region is found near the right edge of the center region, near an area where the center-surround edge contrast is very low. The occlusion model would interpret that pixel and that region as belonging to the occluder, therefore, and would instead select the darkest pixel in the center region as representing the true luminance of the moon. That is, the two models produce conflicting predictions. Notably, the process of identifying occlusion cues is much more effortful on stimuli that are built using cloudy textures and low-contrast centers.

What is most surprising about the inconsistency is that, despite wide acceptance of occlusion as a cue that is used by our visual system, the occlusion model's predictions are inconsistent with Anderson and Winawer's (2005) data. As was demonstrated in the present psychophysical experiment, however, both models are correct, but each model applies to only certain textures. That is, I make the novel prediction here that if a texture has properties that emphasize occlusion information, the occlusion model will give correct predictions. In contrast, if a texture hides occlusion information, the Anderson and Winawer model will give correct predictions. That is, I predict a three-way interaction between texture, center contrast, and average surround luminance that is consistent with the predictions in Table 1.

\section{METHOD}

\section{Participants}

Twelve volunteers, including the author as well as university undergraduate and graduate students, participated. Their vision was normal or corrected to normal. Ten participants were naive with respect to the purpose of the experiment.

\section{Apparatus}

Testing and data collection was done on a $\mathrm{PC}(\mathrm{P} 4,3 \mathrm{GHz})$ that was set to a resolution of $800 \times 600$ pixels and a refresh rate of $75 \mathrm{~Hz}$. Responses were recorded via mouse buttonpresses. Viewing distance was $68.5 \mathrm{~cm}$, at which the stimulus subtended a $16^{\circ} \times 16^{\circ}$ area (not including the response bar; see below).

\section{Stimuli}

Two types of textures were created. The first texture consisted of grayscale noise with a $1 / f^{4}$ power spectrum (cloudy texture), replicating Anderson and Winawer's (2005) method. The second was a two-tone texture, consisting of zeros in its right half, and ones in its left half (or vice versa). The two-tone texture had no intermediate luminance levels and thus contained a single sharp edge that created clear T-junctions where the edge intersected the center-surround boundary. Clear T-junctions are important occlusion cues that help to establish depth relationships; thus, the two-tone texture was expected to produce the results that are most consistent with the occlusion model.

The thresholded texture was not used, because it differed in significant ways from both the cloudy texture and Albert's (2007) own choice of texture, the square-wave grating. Indeed, the thresholded texture (1) is more complex than Albert's square-wave grating, (2) has an energy spectrum that is different from that of both the 
cloudy texture and the square-wave texture, and (3) does not always offer clear T-junctions, unlike the square-wave grating.

These textures $(T)$ were normalized to a range of $0 \ldots 1$ and the luminance range of these textures was then modified independently within a circular center region $(C)$ and its surrounding region $(S)$, according to the following equation:

$$
C_{i j}=T_{i j}\left(C_{\mathrm{A}}-C_{\mathrm{B}}\right)+C_{\mathrm{B}} \text { and } S_{i j}=T_{i j}\left(S_{\mathrm{A}}-S_{\mathrm{B}}\right)+S_{\mathrm{B}},
$$

where $i$ and $j$ indicate position on the image (omitted henceforth for simplicity), and the luminance configuration is defined as the constant luminance values (in cd $/ \mathrm{m}^{2}$ ) of $C_{\mathrm{A}}, C_{\mathrm{B}}, S_{\mathrm{A}}$, and $S_{\mathrm{B}}$. The luminance configuration thus defines the minimum, maximum, and range values for each region (e.g., min value for center $=\min \left(C_{\mathrm{A}}, C_{\mathrm{B}}\right)$; range for center region $\left.=\left|C_{\mathrm{A}}-C_{\mathrm{B}}\right|\right)$. The four constants forming each possible luminance configuration are given in Table 1.

The cloudy textures moved at $2.2^{\circ} / \mathrm{sec}$, at $23.6^{\circ}$ upward and to the left. This motion, over time, reduced the risk that most pixels within the center would be confined to a range of possible values, and it thus produced a variety of edge properties. The two-tone texture did not move.

The luminance values (see Table 1) were selected (1) to provide a better range of possible responses, thus avoiding ceiling and floor effects, and (2) to provide good contrasts at the edges, as judged subjectively. The specific luminance (and the corresponding mean luminance) values that were used are not important here, provided that the stimulus contained the T-junctions. Note that although the average luminance of the center region in low-contrast stimuli also has slightly lower luminance than the center region in high-contrast stimuli, this does not affect the conclusions of the present article.

\section{Procedure}

Participants were presented with a stimulus that had a combination of a given luminance configuration and texture, and they had to report the perceived luminance of the moon on the response bar to the left of the stimulus (see Figure 3). The response bar ranged from white to black. A small white bar indicated the participant's response, which the participants could adjust to their satisfaction using a mouse. They then pressed the space bar to proceed to the next trial. Participants completed practice trials until they felt comfortable with the task. Each condition was repeated five times over the course of the experiment. Participants were informed to respond when they perceived that the moon was opaque and partly occluded, and to wait for that percept if they perceived anything else, such as a semitransparent circle above a textured background. These instructions were consistent with Anderson and Winawer's (2008) methods.

\section{RESULTS}

The conditions in the present experiment included the critical conditions of Anderson and Winawer's (2005; see the present Table 1) experiment, in which the surround texture could be light or dark, and figure contrast could be high or low (see Figure 2), as well as the same four conditions with stimuli that were built using a two-tone texture.

Figure 2 shows the data that were collected, as well as predictions that were generated using the two models. There was a three-way interaction between texture, surround luminance, and figure contrast $[F(1,11)=204$, $p<.0001]$. On average, figures on light surrounds were perceived to be darker than figures on dark surrounds $[F(1,11)=218, p<.0001]$; however, the opposite was true with stimuli that were built using a combination of the low-contrast center and the two-tone textures. At low contrast, the interaction between surround luminance and texture was significant $[F(1,11)=72, p<.0001]$.
Another way to understand the three-way interaction is to decompose the effect with respect to texture. With the cloudy texture (similar to that used by Anderson \& Winawer, 2005; the present Figure 2, left), the figure was always perceived as lighter (or darker) when placed on a darker (or lighter) surround, regardless of whether the figure was high or low contrast. This replicated Anderson and Winawer's (2005) results and is consistent with their model predictions. With the two-tone texture (Figure 2, right), however, there is a clear interaction between figure contrast and surround luminance $[F(1,11)=2,540$, $p<.0001]$. Indeed, the Anderson-Winawer illusion was reversed at the low-contrast range, consistent with the occlusion model.

The effect was so robust that this pattern of results was clear in single-participant data. Indeed, all 12 participants showed the reversal for the low-contrast two-tone texture, and none of them showed a reversal for any of the three other stimuli (i.e., high-contrast two-tone, low-contrast cloudy, high-contrast cloudy). It is thus clear that texture and luminance configuration interacted to determine perceived lightness.

\section{DISCUSSION}

\section{Review of Results}

I have shown via models and empirical data that the Anderson-Winawer illusion cannot be explained simply by occlusion mechanisms. Indeed, the occlusion account produces an opposite pattern of results from that observed by Anderson and Winawer (2005) with low-contrast center stimuli. This has been confirmed through model predictions (see Equations 1 and 2, Table 1, and Figure 2) as well as through a psychophysical experiment with stimuli that were designed to emphasize occlusion cues (i.e., twotone textures).

The occlusion model predicts a reversal of the illusion at low contrast as a result of a change in the occlusion cues. This reversal does not occur with cloudy textures, as was shown in the original data (Anderson \& Winawer, 2005) as well as in the present replication. The reversal does occur when the texture is simplified to a two-tone texture, however, which amplifies occlusion cues by removing extraneous information. The present results thus provide the first demonstration that there are two strategies at work to resolve the problem, with the low-contrast conditions serving as a special case in which the two strategies produce conflicting effects.

The comparison between cloudy and two-tone textures is central to the issue, because both textures contain all of the information that is necessary for either model to make predictions. Using Figure 3 again as an example $\left(C_{\mathrm{A}}=\right.$ $15, C_{\mathrm{B}}=61, S_{\mathrm{A}}=6, S_{\mathrm{B}}=61$; see Table 1, low-dark), the Anderson-Winawer prediction is as follows: (1) The center region is on average lighter than the surround region; (2) thus, the moon will be perceived as lighter than the background; (3) thus, the lightest pixel of the center (with luminance of $C_{\mathrm{B}}=61$ ) will be taken to reflect the moon's lightness. The occlusion model's prediction is as 
follows: (1) The region of lowest center-surround edge contrast is light (see the bottom right of the center region); (2) thus, the occluder will be perceived to be white clouds (with luminance of $S_{\mathrm{B}}=C_{\mathrm{B}}=61$ ); (3) thus, the darkest pixel of the center will be taken to reflect the moon's lightness (with luminance of $C_{\mathrm{A}}=15$ ). Because the two models use different criteria (global and local polarity, respectively), they arrive at different conclusions. In the case of the two-tone texture, the occlusion model wins the competition. In the case of the cloudy texture, however, the Anderson-Winawer prediction wins the competition. It is unclear which model wins the competition in the case of thresholded cloudy textures.

The reversal of the effect also brings into question Anderson and Winawer's $(2005,2008)$ explanation. The Anderson-Winawer prediction was initially based on local edge contrast (similarly to Albert's [2007] model); however, they did not explain how the addition of layering led to the predictive model that they used, and why the reversal of the effect at low figure contrast does not occur.

\section{Related Issues}

There are additional issues worth discussing here. First, the stimuli in Figure 1A and those in Figure 1C appear qualitatively different. The sharp occlusion cues that are present in Figure 1C are difficult to find in Figure 1A, and Figure 1A contains far more gradients than does Figure 1C. Further experiments are necessary to isolate which of these differences accounts for the change in strategy.

Second, the percepts at intermediate levels of figure contrast can be tri-stable for many stimuli (e.g., Figure 1, columns 2-5): The circles can be interpreted as light circles behind a dark occluder, as dark circles behind a light occluder, or even as semitransparent circles floating above a two-tone background. Indeed, all of these percepts are consistent with ambiguous transparency cues (Adelson, 1993; Singh \& Anderson, 2002). An experiment that is currently in progress is measuring the relative frequency of occurrence of these different percepts.

Finally, Anderson and Winawer $(2005,2008)$ also found a smaller bias toward perceiving the moons as lighter than they had predicted on the basis of the lightest or darkest pixel. This bias cannot be due to incomplete lightness constancy, because although it correctly predicts that moons will be lighter than the darkest pixel, it also incorrectly predicts that moons will be darker than the lightest pixel. In fact, the bias likely results from compensation for atmospheric darkening (i.e., the reduction in light intensity when it passes through any semitransparent medium). This indicates that a perceptual mechanism exists to correct for this, and it triggers when a surface is perceived as partially occluded everywhere, such as when it is behind any semitransparent surface. Neither the occlusion model nor the Anderson and Winawer model account for this effect.

\section{Conclusion}

In summary, the Anderson-Winawer illusion at low figure contrast is in the opposite direction from that predicted by occlusion mechanisms such as Albert's (2007), which has been confirmed by modeling and a psychophysical experiment. In view of this important difference, I conclude that Albert's explanation has little bearing on the nature of the Anderson-Winawer illusion, and it should therefore be revised or rejected. Anderson and Winawer's (2005) model also lacks important details. Indeed, it is not clear how their predictions could arise from layering.

I propose a novel model to reconcile these differences. This model classifies the stimuli according to one criterion; once classified, the stimuli are interpreted with appropriate mechanisms.

The model works only on stimuli in which the texture that is present in the center region is similar and continuous with the texture that is present in the surround region. This is the case in all of the stimuli that are presented in Figure 1. If the texture is dissimilar, or if there is a discontinuity at the center-surround edge, then the texture in the center region will be perceived as belonging to the figure, and the model will not apply. This occurs, for example, when the center texture is rotated or contrast-inverted (not shown), as Albert (2007) and Anderson and Winawer (2005) used in their control conditions. If the textures are perceived to be similar and continuous across center and surround regions, however, the model that is described below will apply.

The model assesses whether there are salient occlusion cues. In the presence of salient occlusion cues (as is the case in Figure 1C, columns 1 and 6), the moon's perceived lightness will be based on the visible parts of the moon, consistent with the occlusion model (Equation 2).

In the absence of salient occlusion cues (i.e., either T-junctions are absent or they are too noisy or blurred to be extracted and used efficiently by the perceptual system), however, the model must rely on simpler cues. This is the case in all of the stimuli that are in Figure 1A. It is unclear at this point what that mechanism is. What is clear is what that mechanism does. It estimates lightness of the moon in three steps: (1) It determines whether the center region is lighter or darker than the surround region, possibly on the basis of average or median luminance, (2) it determines the luminance range that is contained within the center region, and (3) it biases the perceived object's lightness close to the most extreme value that is contained in that luminance range, consistent with the direction that was determined in the first step (e.g., close to the lightest or darkest pixel in that region if the center region is on average lighter or darker, respectively, than the surround region). These three steps produce predictions that are consistent with Anderson and Winawer's (2005; the present Equation 1) model and with data that were collected on stimuli that were built with cloudy textures (Anderson \& Winawer, 2005; the present study). I suggest that the mechanism that is involved here is a computationally simpler variant of the occlusion mechanism, operating on a scale that is larger than that of the occlusion cues. This mechanism correctly detects the presence of variable opacity cues, thus signaling the presence of an occluder; however, the failure to encode accurately the borderownership relations leaves the stimulus ambiguous. This mechanism resolves the ambiguity by comparing average center and surround luminance values. 
The model that is presented here is an improvement on both Anderson and Winawer's (2005) model and Albert's (2007) model because it accounts for the texture dependency of perceived lightness.

Failure to incorporate this novel strategy in lightness theories and scene-interpretation algorithms will result in interpretations of corresponding scenes that are inconsistent with perception.

\section{AUTHOR NOTE}

Portions of this article were presented at CVR 2005 in Toronto, ON, and at VSS 2008 in Naples, FL. This research was supported by NSERC Grant OP227224 to Hugh R. Wilson and a CIHR Grant to Martin Arguin, Frédéric Gosselin, and Dan Bub. I thank Hugh R. Wilson, Frédéric Gosselin, Martin Arguin, Charlie Chubb, and both reviewers for comments on earlier versions of this article. Correspondence concerning this article should be addressed to F. J. A. M. Poirier, Centre de Recherche en Neuropsychologie et Cognition, Université de Montréal, C.P. 6128, succursale Centre-Ville, Montréal, QC, H3C 3J7 Canada (e-mail: frederic .poirier@umontreal.ca).

\section{REFERENCES}

Adelson, E. H. (1993). Perceptual organization and the judgment of brightness. Science, 262, 2042-2044. doi:10.1126/science.8266102

AdELSON, E. H. (2000). Lightness perception and lightness illusions. In M. Gazzaniga (Ed.), The new cognitive neurosciences (2nd ed., pp. 339-351). Cambridge, MA: MIT Press.

Albert, M. K. (2007). Occlusion, transparency, and lightness. Vision Research, 47, 3061-3069. doi:10.1016/j.visres.2007.06.004

Anderson, B. L. (1997). A theory of illusory lightness and transparency in monocular and binocular images: The role of contour junctions. Perception, 26, 419-453. doi:10.1068/p260419

ANDERSON, B. L., \& WinAwER, J. (2005). Image segmentation and lightness perception. Nature, 434, 79-83. doi:10.1038/nature03271

Anderson, B. L., \& Winawer, J. (2008). Layered image representations and the computation of surface lightness. Journal of Vision, 8(7, Art. 18), 1-22. doi:10.1167/8.7.18

Fine, I., MacLeod, D. I. A., \& Boynton, G. M. (2003). Surface segmentation based on the luminance and color statistics of natural scenes. Journal of the Optical Society of America A, 20, 1283-1291. doi:10.1364/JOSAA.20.001283

HeinemanN, E. G. (1955). Simultaneous brightness induction as a function of inducing- and test-field luminances. Journal of Experimental Psychology, 50, 89-96.

Horeman, H. W. (1963). Inductive lightness depression as influenced by configurational conditions. Vision Research, 3, 121-130.

Kelly, F., \& Grossberg, S. (2000). Neural dynamics of 3-D surface perception: Figure-ground separation and lightness perception. Perception \& Psychophysics, 62, 1596-1618.

LI, Z. (2000). Pre-attentive segmentation in the primary visual cortex. Spatial Vision, 13, 25-50. doi:10.1163/156856800741009

Singh, M., \& ANDERSON, B. L. (2002). Toward a perceptual theory of transparency. Psychological Review, 109, 492-519. doi:10.1037/0033 $-295 X .109 .3 .492$

White, M. (1979). A new effect of pattern on perceived lightness. Perception, 8, 413-416. doi:10.1068/p080413

White, M. (1981). The effect of the nature of the surround on the perceived lightness of grey bars within square-wave test gratings. Perception, 10, 215-230. doi:10.1068/p100215

(Manuscript received August 13, 2008; revision accepted for publication April 6, 2009.) 